\section{The increase of} aqueous tear volume by diquafosol sodium in dry-eye patients with Sjögren's syndrome: a pilot study

\author{
Abstract \\ Purpose To investigate the effect of 3\% \\ diquafosol sodium ophthalmic solution \\ (DQS) on aqueous tear volume increase \\ in dry-eye patients with Sjögren's \\ syndrome (SS). \\ Methods In this pilot study, 17 dry-eye \\ patients with SS (1 male and 16 females; \\ mean age: 66.4 years) were enrolled and \\ underwent topical instillation of two \\ ophthalmic solutions, artificial tears (AT) \\ in one eye and DQS in the fellow eye, in \\ a masked manner. The central lower tear \\ meniscus radius (TMR) curvature was \\ measured before and at $\mathbf{1 5} \mathrm{min}$ after \\ instillation by video-meniscometry. \\ Simultaneously, all patients self-evaluated \\ their symptoms of wetness and stinging using \\ a visual analog scale (VAS, in millimeters). \\ Results Topical instillation of DQS \\ significantly increased the TMR at $15 \mathrm{~min}$ \\ (mean: $0.21 \pm 0.08$ (SD) $\mathrm{mm}$ ) compared with \\ at baseline (mean: $0.16 \pm 0.07 \mathrm{~mm})(P<0.001$, \\ paired $t$-test), whereas AT had no effect at \\ baseline (mean: $0.18 \pm 0.09 \mathrm{~mm}$ ) or at $15 \mathrm{~min}$ \\ (mean: $0.18 \pm 0.09 \mathrm{~mm}$ ). The visual VAS score \\ of wetness at $15-\mathrm{min}$ post-instillation \\ increased in both groups compared with at \\ baseline. In the DQS-treated eyes, the post- \\ instillation change in TMR from baseline was \\ not correlated with the baseline value of the \\ Schirmer test, corneal staining score, or \\ conjunctival staining score. \\ Conclusions Topical instillation of DQS \\ increased aqueous tear volume on the ocular \\ surface of dry-eye patients with SS, with its \\ action being independent of lacrimal gland \\ function. \\ Eye (2016) 30, 857-864; doi:10.1038/eye.2016.54; \\ published online 8 April 2016
}

N Yokoi, H Kato and S Kinoshita

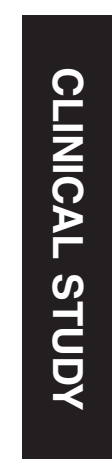

Introduction

According to the Dry Eye WorkShop (DEWS) definition, dry eye is a multifactorial disease of the tears and ocular surface that results in symptoms of discomfort, visual disturbance, and tear-film instability with potential damage to the ocular surface. It is accompanied by increased tear-film osmolarity and inflammation of the ocular surface. ${ }^{1}$ In addition, the DEWS definition classifies dry eye as either aqueous tear-deficient dry eye or evaporative dry eye. Aqueous teardeficient dry eye is the type of dry eye typically observed in Sjögren's syndrome (SS) cases. SS is an organ-specific autoimmune disease ${ }^{2}$ and is considered to be associated with decreased tear-fluid secretion from the lacrimal gland and inflammation at the lacrimal gland and the ocular surface. ${ }^{3-5}$

Diquafosol sodium (DQS) ophthalmic solution, which has been approved in Japan for the treatment of dry eye, increases fluid secretion, ${ }^{6-8}$ mucin secretion, ${ }^{9,10}$ and membranebinding mucin gene expression on the corneal surface. ${ }^{11}$ Reportedly, DQS increases tear-film stability on the ocular surface, thereby improving the subjective symptoms and objective signs of dry eye. ${ }^{12-15}$ In addition, DQS exhibits $\mathrm{P}_{2} \mathrm{Y}_{2}$ receptor agonist activity and accelerates fluid transport from the serosal to mucosal (tear) side via chloride channel activation following intracellular calcium ion concentration elevation in the conjunctival epithelium. ${ }^{16,17}$ The findings of a previous study suggested that the stimulatory action of DQS on fluid secretion is independent of lacrimal gland function, because the Schirmer test results for a rat model of lacrimal gland removal showed aqueous fluid secretion. ${ }^{6}$
Department of Ophthalmology, Kyoto Prefectural University of Medicine, Kyoto, Japan

\section{Correspondence:} $\mathrm{N}$ Yokoi, Department of Ophthalmology, Kyoto Prefectural University of Medicine, 465 Kajii-cho, Hirokoji-agaru, Kawaramachi-dori, Kamigyo-ku, Kyoto 6020841, Japan

Tel: +81752515578; Fax: +81752515663 E-mail: nyokoi@koto.kpu-m. ac.jp

Received: 30 June 2015 Accepted in revised form: 11 February 2016 Published online: 8 April 2016 
Reflective meniscometry is a method used to optically measure the tear meniscus radius (TMR). ${ }^{18-21}$ In a previous study, we measured the changes in TMR over time in normal human eyes after instillation of DQS using meniscometry, and reported that there was a significant increase in the fluid volume up until $30 \mathrm{~min}$ after the instillation. ${ }^{8}$ In this pilot study, we used meniscometry to evaluate the changes in aqueous tear volume after instillation of DQS in patients for whom a definitive diagnosis of SS with dry eye (determined by a Schirmer 1 test value of $<10 \mathrm{~mm}$ ) had been provided, and compared the results with those obtained after instillation of an artificial tear (AT) solution.

\section{Materials and methods}

\section{Subjects}

Approval was obtained from the Institutional Review Board of Kyoto Prefectural University of Medicine, Kyoto, Japan prior to the initiation of this study. The research was conducted in compliance with the Tenets set forth in the Declaration of Helsinki, and prior written informed consent was obtained from all subjects after receiving a detailed explanation of the nature of the study and possible consequences associated with participation in the study. This study is a prospective, randomized, double-masked, comparative study of 17 subjects ( 1 male and 16 females; mean age: $66.4 \pm 10.1$ (SD) years) who were diagnosed with SS by Fox's criteria ${ }^{22}$ were enrolled. Subjects who had a Schirmer 1 test value of $\geq 10 \mathrm{~mm}$ in either eyes and who were judged inappropriate for this study by doctors were excluded. There were no patients with secondary SS.

\section{Tear function tests and ocular surface evaluation}

In each patient, after obtaining the baseline measurement of the TMR by video-meniscometry, ophthalmic examinations including measurement of the fluorescein break-up time (FBUT), and assessments of corneal and conjunctival fluorescein staining were performed. For the measurement of FBUT and evaluation of fluorescein staining, a slit-lamp biomicroscope with a cobalt blue filter and blue-free filter ${ }^{23}$ was used. After two drops of saline solution were instilled onto a fluorescein test strip (Showayakuhinkako Co., Tokyo, Japan), the strip was shaken vigorously. In order not to inadvertently increase the patient's tear volume, strict attention was paid to gently touch the strip to the center of the lid margin to stain the tear film with fluorescein, which was then followed by several natural blinks. Subsequently, the FBUT was measured as the time (in s) until the first appearance of a dark spot in the precorneal tear film when the eye was kept open. The FBUT was measured by stopwatch three times and then averaged. The FBUT evaluation was followed by the assessments of fluorescein staining of the ocular surface epithelium. For assessment of corneal epithelial fluorescein staining, the cornea was divided into five portions. The staining was scored from 0 to 3 for each portion and the total score was then calculated. ${ }^{24}$ For assessment of conjunctival epithelial fluorescein staining, the bulbar conjunctiva was divided into nasal or temporal portions. The van Bijsterveld scoring system ${ }^{25}$ was used to score the conjunctival epithelial damage from 0 to 3 in each portion, and the total score was then calculated. Accordingly, the overall corneal and conjunctival epithelial damages were scored on scales of 0-15 and 0-6 points, respectively. In this study, the Schirmer test without topical anesthesia (ie, the Schirmer 1 test) was performed using standard Schirmer test strips (Showayakuhinkako). The strips were placed for $5 \mathrm{~min}$ at the temporal one-third of the lower conjunctival fornix in both eyes of each patient and then removed. The length (in millimeters) of filter paper that had been wetted was then recorded. To avoid any influence of the test eye-drop instillation on the Schirmer 1 test values, the Schirmer 1 test values at the last visit were used for each patient.

\section{Evaluation of aqueous tear volume changes by meniscometry}

To avoid the influence of ocular surface staining, the evaluation of tear volume changes was performed at $>10$ min after the evaluation of fluorescein staining of the cornea and conjunctiva. Each of the 17 patients received a single drop of 3\% DQS ophthalmic solution (Diquas; Santen Pharmaceutical Co. Ltd., Osaka, Japan) in one eye and a single drop of the AT solution (Soft Santear; Santen Pharmaceutical) in the fellow eye. These two ophthalmic solutions were randomly assigned to the right or left eye for each subject, and investigators remained masked and treatment of each subject's eyes remained unidentified throughout the study. The central lower TMR was measured via video-meniscometry ${ }^{19-21,26}$ as an index of the total aqueous tear volume over the ocular surface. ${ }^{21}$ In brief, an illuminated target comprising a series of horizontal stripes was projected via meniscometry onto the central lower tear meniscus, and the specular reflex image of the target was then recorded by using a digital video recorder. Image analysis software was used to calculate the TMR by application of a concave mirror formula. Recordings of the target image were performed at baseline (pre-instillation) and at $15 \mathrm{~min}$ after the instillation of each single eye drop. In addition, subjective ocular symptoms (ie, wetness or stinging) were recorded by the subjects using a $100-\mathrm{mm}$ visual 
analog scale (VAS). The calculated TMR and the subjective ocular symptom levels were then compared with the baseline values at $15 \mathrm{~min}$ after instillation in both the DQS- and AT-treated eyes. The change in TMR $(\Delta \mathrm{TMR}=\mathrm{TMR}$ at $15 \mathrm{~min}-$ baseline TMR, $\mathrm{mm})$ was calculated for each eye. Moreover, for the purpose of determining the relationships among lacrimal gland function, epithelial damage on the ocular surface, and increased aqueous tear volume after the instillation of DQS, the correlations among the Schirmer 1 test values, baseline corneal and conjunctival damage scores, and $\triangle T M R$ in the DQS-treated eyes were investigated.

\section{Statistical analysis}

Paired $t$-tests were used for statistical comparisons of the data including TMR, Schirmer 1 test value, and FBUT. Wilcoxon signed-rank test were used for ocular surface staining scores and VAS scores. Pearson's correlation coefficient was used to assess the correlative relationships between $\Delta \mathrm{TMR}$ and the baseline Schirmer 1 test value, and corneal and conjunctival fluorescein staining scores. A $P$-value of $<0.05$ was considered statistically significant.

\section{Results}

The baseline Schirmer 1 test values in the AT- and DQS-treated eyes at the last visit, TMR, FBUT, and corneal and conjunctival fluorescein staining scores are shown in Table 1. There were no significant differences between the AT- and DQS-treated eyes with respect to these values.

The baseline TMR was $0.18 \pm 0.09 \mathrm{~mm}$ in the AT-treated eyes and $0.16 \pm 0.07 \mathrm{~mm}$ in the DQS-treated eyes. The TMR in the AT-treated eyes at $15 \mathrm{~min}$ after instillation was $0.18 \pm 0.09 \mathrm{~mm}$, showing no significant difference from the baseline value ( $P=0.4575$, paired $t$-test) (Figure 1). However, the TMR in the DQS-treated eyes at $15 \mathrm{~min}$ after instillation was $0.21 \pm 0.08 \mathrm{~mm}$, which was significantly higher than the baseline value and reflected a significant increase in the aqueous tear volume on the ocular surface $(P<0.0001$, paired $t$-test) (Figure 1$)$. Representative meniscometry images taken of the central lower tear meniscus at baseline and at $15 \mathrm{~min}$ after instillation of AT or DQS are shown in Figure 2.

The baseline VAS scores of wetness in AT- and DQS-treated eyes were $11.6 \pm 11.1$ and $12.9 \pm 14.2$, respectively. The baseline scores of stinging in ATand DQS-treated eyes were $31.8 \pm 26.9$ and $30.6 \pm 30.1$, respectively. The VAS scores of subjective symptoms at 15 min after instillation revealed that the score of wetness was significantly increased from baseline in both the AT- and DQS- treated eyes $(36.1 \pm 23.2$ and $36.9 \pm 19.0$, respectively, $P<0.001$, Wilcoxon signed-rank test) (Figure 3a). Conversely, the score of stinging tended to decrease from baseline in both the AT- and DQS-treated eyes, and there was a significant difference between the baseline and post-instillation scores in the AT-treated eyes $(11.8 \pm 16.6, P<0.01$, Wilcoxon signed-rank test) (Figure 3b).

Moreover, the relationships between $\triangle \mathrm{TMR}$ value and the baseline parameters were evaluated in the DQStreated eyes, and there was no significant correlation between each baseline value and $\Delta$ TMR value (Figure 4).

\section{Discussion}

SS is an organ-specific autoimmune disease in which destruction of the lacrimal glands caused by lymphocytic infiltration leads to decreased lacrimal gland function and results in aqueous tear-deficient dry eye. Decreased function of the lacrimal glands in SS patients is thought

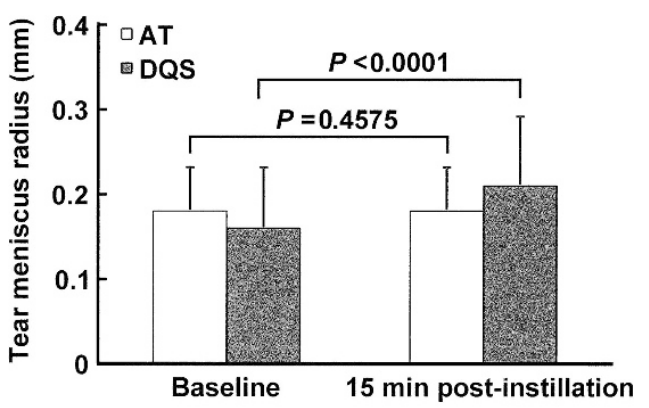

Figure 1 Changes in the tear meniscus radius (TMR) at baseline and at $15 \mathrm{~min}$ after instillation of an artificial tear (AT) solution and diquafosol sodium (DQS) ophthalmic solution. The data represent mean \pm SD. $P$-values were calculated by use of the paired $t$-test.

Table 1 Baseline demographic data for AT- and DQS-treated groups

\begin{tabular}{lccccc}
\hline & Schirmer 1 test $(\mathrm{mm})$ & TMR $(\mathrm{mm})$ & FBUT (seconds) & Fluorescein score (cornea) (0-15) & Fluorescein score (conjunctiva) (0-6) \\
\hline AT & $3.2 \pm 2.5$ & $0.18 \pm 0.09$ & $1.5 \pm 1.1$ & $7.1 \pm 3.0$ & $5.4 \pm 1.0$ \\
DQS & $3.6 \pm 2.4$ & $0.16 \pm 0.07$ & $1.4 \pm 1.3$ & $5.8 \pm 3.6$ & $5.2 \pm 1.1$ \\
$P$-value & 0.511 & 0.225 & 0.928 & 0.226 & 0.281 \\
\hline
\end{tabular}

Abbreviations: AT, artificial tear solution; DQS, 3\% diquafosol sodium ophthalmic solution; FBUT, fluorescein break-up time; TMR, tear meniscus radius. Data values are mean $\pm \mathrm{SD}$. $P$-values were calculated by use of the Paired $t$-test for Schirmer 1 test, TMR, and FBUT, and by use of Wilcoxon signed-rank test for fluorescein scores. 

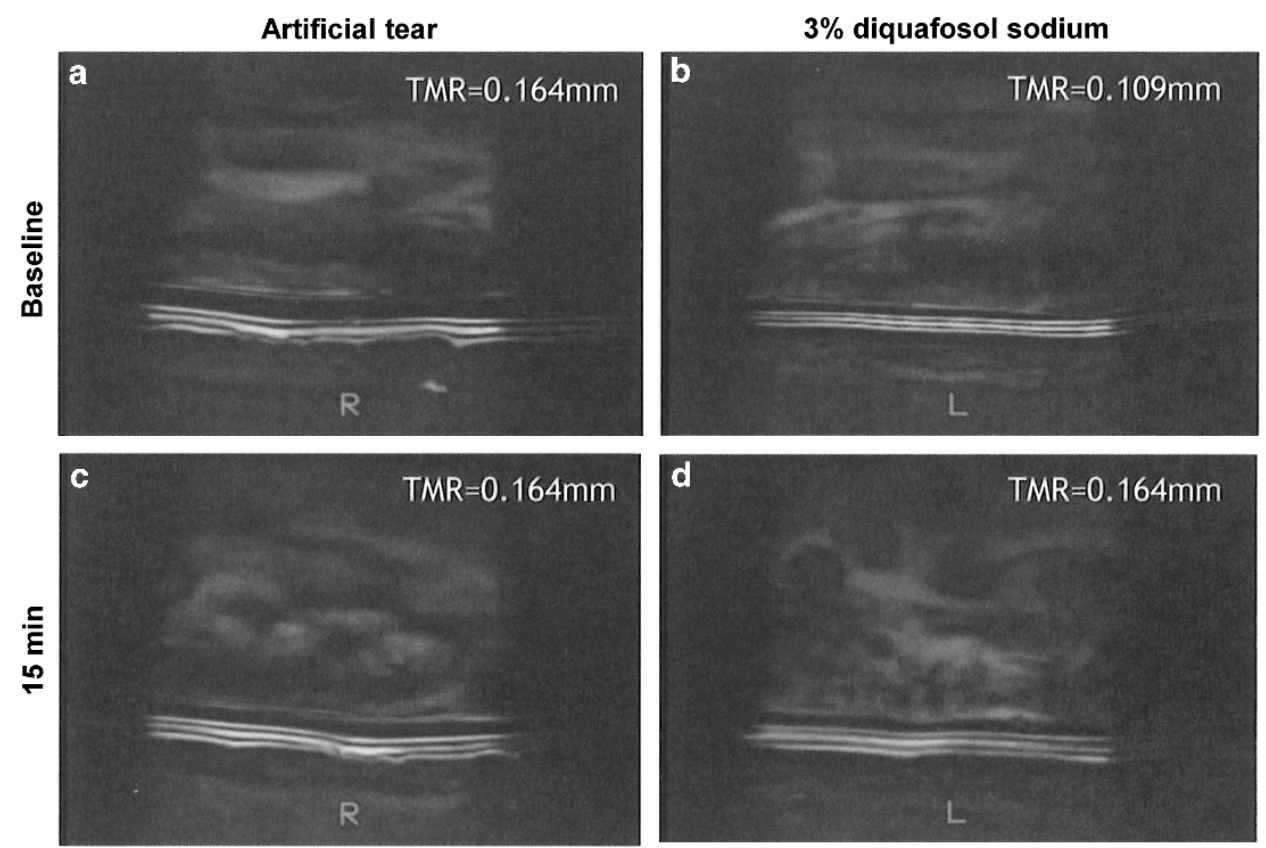

Figure 2 Representative images of the central lower tear meniscus of two eyes of one patient via meniscometry at baseline and at 15 min after instillation of AT or DQS. The images show the changes at baseline (a) and at $15 \mathrm{~min}$ after AT instillation, (c) and at baseline (b) and at 15 min after instillation of DQS (d).
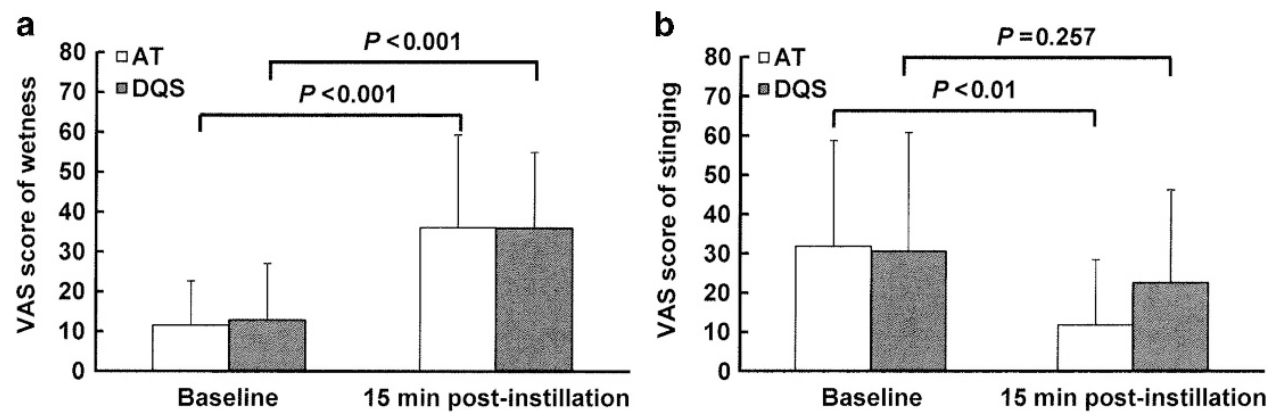

Figure 3 Subjective changes in ocular comfort (that is, wetness and stinging) at 15 min after instillation of AT or DQS. The patientassessed values of ocular comfort for wetness (a) and stinging $(\mathbf{b})$ were recorded using a 100-mm visual analog scale (VAS). The data represent mean \pm SD. $P$-values were calculated by use of the Wilcoxon signed-rank test.

to depend on the degree of lymphocytic infiltration, and lymphocytic infiltration into the lacrimal glands is more closely related to Schirmer test values with nasal stimulation than Schirmer 1 test values. ${ }^{27}$ In addition, ocular surface disorder has been reported to be more closely correlated with Schirmer test values with nasal stimulation. ${ }^{3,28}$ Although the SS patients enrolled in this present study did not have Schirmer test values with nasal stimulation, the Schirmer 1 test values were $<10 \mathrm{~mm}$, and the baseline TMR values evaluated via meniscometry on the examination day were relatively low (mean: $0.160 \mathrm{~mm}$ ). This mean TMR was $\sim 50 \%$ of our previously reported mean TMR $(0.30 \mathrm{~mm})$ in normal eyes, ${ }^{26}$ suggesting that the enrolled patients had lacrimal gland function that was sufficiently poor for the evaluation in this study. Furthermore, as only one male was assessed in the current study, this gender disparity would skew the results. However, it is difficult to enroll same number of male SS patients to female patients because SS is a femaledominant disease. Further investigation may be necessary but the male patient responded to DQS in the present study.

Previous studies using optical coherence tomography (OCT) reported that the tear volumes in SS dry eyes were significantly lower than those in normal eyes or non-SS dry eyes. ${ }^{29-31}$ In the present study, we used meniscometry to measure changes in tear volume, because it appeared difficult to position the window for meniscus measurement with good reproducibility using OCT, and OCT might therefore be inappropriate as a means for analyzing 


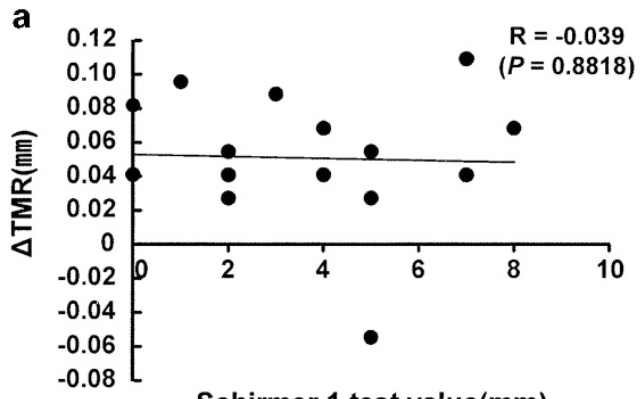

Schirmer 1 test value(mm)
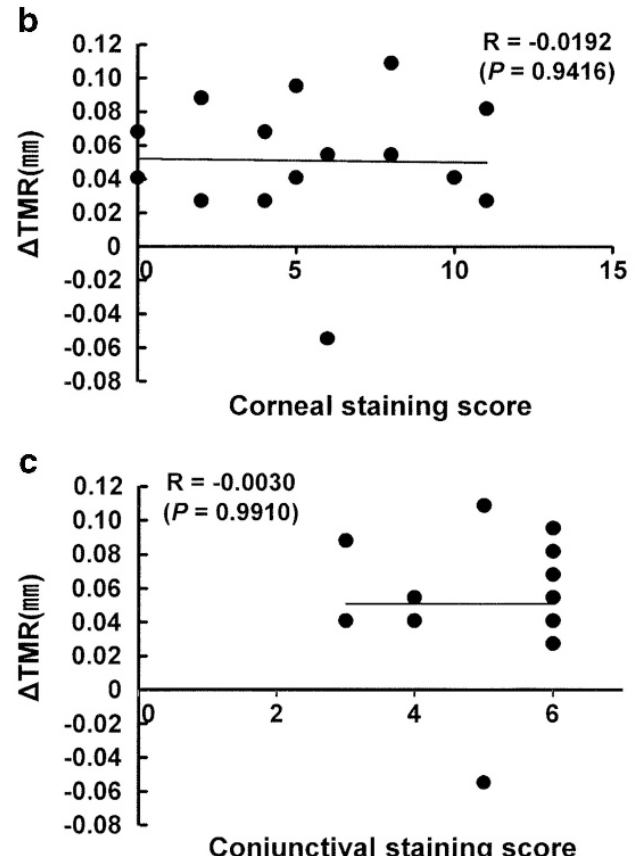

Figure 4 Correlative analyses between the $\triangle \mathrm{TMR}$ and ocular signs in the DQS treatment. Pearson's correlation coefficient was calculated for correlations between $\triangle \mathrm{TMR}$ and Schirmer 1 test value, (a) corneal fluorescein staining score, (b) and conjunctival fluorescein staining score (c) in the DQS-treated eyes.

meniscus parameters over time. In addition, we previously reported a primary correlation between the TMR and aqueous tear volume on the ocular surface in individual eyes when measured by meniscometry, ${ }^{26}$ and that correlation has not been proven by OCT. This is because TMR measurement by meniscometry is considered to be more useful for monitoring the time course of aqueous tear volume on the ocular surface after eye-drop instillation. Meniscometry allows for a non-invasive measurement of a very low meniscus with a TMR of, for example, $0.05 \mathrm{~mm},{ }^{19}$ and observation of a meniscus with a specific working distance $(24.0 \mathrm{~mm})$ by projecting white light from the front of the meniscometer onto the bottom of the meniscus with an anterior, concave surface, thus allowing follow-up of the time course of the meniscus changes with good reproducibility. Therefore, meniscometry is considered to be especially useful for evaluating cases of SS dry eyes with lower aqueous tear volume, such as those in the patients enrolled in this present study.

As reported by Fujihara et al, ${ }^{6}$ DQS produced a stimulatory action on fluid secretion in a rat model of exorbital lacrimal gland removal, with the model rats demonstrating little difference from normal rats in the increase in aqueous tear volume on the ocular surface observed in a 1-min Schirmer test. Those results show that the stimulatory action of DQS on fluid secretion is independent of the exorbital lacrimal glands in animals. In normal human eyes evaluated by meniscometry, the increase in TMR reportedly reached a peak at 10-15 min after the instillation of DQS ophthalmic solution, ${ }^{8}$ as with the findings in the animal studies. Therefore, in this present study investigating the effect on the fluid increase in SS dry eyes, the increase in TMR at 15 min after instillation was evaluated. The results showed that the mean $\triangle T M R$ at 15 min after DQS instillation in SS patients was $0.051 \pm 0.037 \mathrm{~mm}$, which was similar to the value in a previous study on healthy subjects $(0.037 \pm 0.019 \mathrm{~mm}){ }^{8}$ Although the $\triangle \mathrm{TMR}$ value is appropriate for evaluation of an increase in aqueous tear volume over time on the same ocular surface, it is impossible to compare the change in radius values of different eyes. ${ }^{18}$ Nonetheless, it is very interesting that the increase in aqueous tear volume after instillation of DQS was similar in healthy subjects and SS patients with decreased lacrimal gland function. Moreover, the results of this present study showed that there was no significant correlation between the $\triangle \mathrm{TMR}$ and Schirmer 1 test values, which reflect reflex tear secretion from the lacrimal glands, and no significant difference from those in the AT-treated eyes, even in patients with ocular irritation considered to be the trigger of reflex tear secretion. These findings suggest that DQS can also increase fluid secretion in human conjunctival epithelial cells, independent of the lacrimal glands.

Reportedly, the $\mathrm{P}_{2} \mathrm{Y}_{2}$ receptor is expressed in conjunctival epithelial cells and goblet cells. ${ }^{32}$ However, there has yet to be a report regarding the changes in $\mathrm{P}_{2} \mathrm{Y}_{2}$ receptor expression in the conjunctival epithelium of SS patients. One of the characteristics of the conjunctival epithelium in SS patients with dry eye is that more severe dry-eye damage is observed compared with that of nonSS patients. ${ }^{33}$ Various abnormalities, such as increased expression levels of inflammatory cytokines and their genes, decreased goblet cell density, and stratification and squamous metaplasia of the conjunctival epithelium have been reported in SS patients, ${ }^{4,34-37}$ thus confirming that $\mathrm{P}_{2} \mathrm{Y}_{2}$ receptor expression changes in the conjunctival epithelium of those patients. However, there was no correlation between $\triangle \mathrm{TMR}$ and the conjunctival staining scores reflecting bulbar conjunctival epithelial damage. In other words, the fluid secretion induced by DQS has 
the potential to be sufficiently effective for the palpebral conjunctival epithelium, regardless of the severity of the bulbar conjunctival epithelial damage, given that there is usually no damage to the palpebral conjunctival epithelium even in patients with severe SS with severe bulbar conjunctival epithelial damage (unpublished data). Moreover, as the $\triangle \mathrm{TMR}$ after the instillation of DQS showed little difference between the eyes of SS patients in this present study and normal eyes, ${ }^{8}$ it should also be considered that there was no change in responsiveness to the stimulatory action of DQS on fluid secretion, even in conjunctival epithelium that has been damaged to some degree. However, if there is extensive and severe epithelial damage in the bulbar and palpebral conjunctivas, DQS may be less effective. It remains to be shown whether DQS is effective in increasing fluid secretion even in eyes with extensively damaged palpebral conjunctiva together with damaged bulbar conjunctiva. One of the limitations of this pilot study was we were unable to check the objective parameters in conjunctival epithelium, such as impression cytology for metaplasia, inflammation markers, and so on. Thus, in addition to male patients, further detailed investigation on the objective parameters and subjective symptom score in a larger scale study is needed.

In this present study, the $\triangle T M R$ at 15 min after instillation was significantly higher in the DQS-treated eyes than in the AT-treated eyes, whereas there was no significant difference between the VAS scores of wetness. We theorize that this was due to the AT increasing the aqueous tear volume on the ocular surface for as long as $5 \mathrm{~min}, 8,26$ thereby maintaining the wetness and making it impossible to detect a difference between the VAS scores despite the significant difference in aqueous tear volume at $15 \mathrm{~min}$ after instillation. Therefore, it would probably have been possible to detect wetness if the eyes were compared even after $30 \mathrm{~min}$, because DQS reportedly significantly increased aqueous tear volume in normal eyes for $30 \mathrm{~min}$ after instillation. ${ }^{8}$ Interestingly, VAS score of stinging was decreased in AT-treated eyes but not in DQS-treated eyes despite of increased tear volume. At present this discrepancy is not clarified. Temporal increased tear volume might improve the eye stinging rather than continuous tear volume increase. There would be different result at $>30 \mathrm{~min}$ after instillation.

Topical instillation of DQS ophthalmic solution is reportedly effective for various types of dry eye, including aqueous tear-deficient dry eye ${ }^{38}$ with a Schirmer test value of $\leq 5 \mathrm{~mm}$ (eg, SS), short BUT type dry eye, ${ }^{39}$ dry eye after surgery, ${ }^{40-42}$ and obstructive meibomian gland dysfunction. ${ }^{43}$ The possible mechanism of that action is that DQS ophthalmic solution increases tear-film stability, thus eliminating the vicious cycle (the core mechanism of dry eye on the ocular surface) by increasing fluid secretion, mucin secretion, and membrane-binding mucin gene expression. ${ }^{13}$ On the ocular surface of healthy subjects evaluated by meniscometry, an AT reportedly increased fluid volume for $\sim 2$ min after instillation, and sodium hyaluronate increased the fluid volume for $\sim 5 \mathrm{~min} .{ }^{18}$ The fluid volume at $15 \mathrm{~min}$ after instillation of DQS was increased in the SS patients with dry eye and decreased lacrimal gland function. This longer mode of action may contribute to protect the ocular surface from the desiccation and ameliorate the epithelial damage. Recently, prolonged use of a visual display terminal has been suggested to cause a decrease in lacrimal gland function. ${ }^{44}$ DQS increases fluid secretion for a certain period of time, independent of the lacrimal glands, by acting on the conjunctival epithelium, and it also makes the ocular surface more hydrophilic by increasing both mucin secretion and membrane-binding mucin expression. Taken together, DQS is considered to be effective for evaporative dry eye associated with visual display terminal work, as well as for aqueous tear-deficient dry eye, including SS dry eye.

In conclusion, DQS ophthalmic solution has been shown to increase aqueous tear volume on the ocular surface by meniscometry in dry-eye patients with SS with decreased lacrimal gland function. In addition, the results suggest that the stimulatory action of DQS on fluid secretion was independent of lacrimal gland function. Thus, DQS is expected to be an effective treatment for patients with aqueous tear-deficient dry eye with decreased lacrimal gland function, including SS dry eye.

\section{Summary}

\section{What was known before}

- Diquafosol facilitates aqueous tear secretion in normal eyes.

What this study adds

- Diquafosol facilitates aqueous tear secretion in dry eyes with Sjögren's syndrome with decreased lacrimal gland function, independent of lacrimal gland function.

\section{Conflict of interest}

The authors declare no conflict of interest.

\section{Acknowledgements}

We wish to thank Mr John Bush (Senior Editor, Kyoto Prefectural University of Medicine) for reviewing the manuscript. We make the following disclosures: Norihiko Yokoi: consultant for Kissei Co., Ltd., Nagano, Japan and Rohto Co., Ltd, Osaka, Japan. Shigeru Kinoshita: consultant for Santen Pharmaceutical Co., Ltd, Osaka, 
Japan and Otsuka Pharmaceutical Co., Ltd., Tokyo, Japan. Hiroaki Kato has no relationship to disclose. This study was supported in part by Grants-in-Aid for scientific research from the Japanese Ministry of Education, Culture, Sports, Science and Technology. The authors have no proprietary or commercial interests in any of the materials discussed in this article.

\section{References}

1 The definition and classification of dry eye disease: report of the Definition and Classification Subcommittee of the International Dry Eye Work Shop. Ocul Surf 2007; 5: 75-92.

2 Fox RI. Sjögren's syndrome. Lancet 2005; 366: 321-331.

3 Tsubota K, Toda I, Yagi Y, Ogawa Y, Ono M, Yoshino K. Three different types of dry eye syndrome. Cornea 1994; 13: 202-209.

4 Kawasaki S, Kawamoto S, Yokoi N, Connon C, Minesaki Y, Kinoshita $\mathrm{S}$ et al. Up-regulated gene expression in the conjunctival epithelium of patients with Sjögren's syndrome. Exp Eye Res 2003; 77: 17-26.

5 Stern ME, Pflugfelder SC. Inflammation in dry eye. Ocul Surf 2004; 2: 124-130.

6 Fujihara T, Murakami T, Fujita H, Nakamura M, Nakata K. Improvement of corneal barrier function by the $\mathrm{P}_{2} \mathrm{Y}_{2}$ agonist INS365 in a rat dry eye model. Invest Ophthalmol Vis Sci 2001; 42: 96-100.

7 Murakami T, Fujita H, Fujihara T, Nakamura M, Nakata K. Novel noninvasive sensitive determination of tear volume changes in normal cats. Ophthalmic Res 2002; 34: 371-374.

8 Yokoi N, Kato H, Kinoshita S. Facilitation of tear fluid secretion by $3 \%$ diquafosol ophthalmic solution in normal human eyes. Am J Ophthalmol 2014; 157: 85-92.

9 Fujihara T, Murakami T, Nagano T, Nakamura M, Nakata K. INS365 suppresses loss of corneal epithelial integrity by secretion of mucin-like glycoprotein in a rabbit short-term dry eye model. J Ocul Pharmacol Ther 2002; 18: 363-370.

10 Takaoka-Shichijo Y, Sakamoto A, Nakamura M. Effect of diquafosol tetrasodium on MUC5AC secretion by rabbit conjunctival tissues. Atarashii Ganka (Journal of the Eye) 2011; 28: 261-265.

11 Takaoka-Shichijyo Y, Nakamura M. Stimulatory effect of diquafosol tetrasodium on the expression of membranebinding mucin genes in cultured human corneal epithelial cells. Atarashii Ganka (Journal of the Eye) 2011; 28: 425-429.

12 Matsumoto Y, Ohashi Y, Watanabe H, Tsubota K. Efficacy and safety of diquafosol ophthalmic solution in patients with dry eye syndrome: a Japanese phase 2 clinical trial. Ophthalmology 2012; 119: 1954-1960.

13 Nakamura M, Imanaka T, Sakamoto A. Diquafosol ophthalmic solution for dry eye treatment. Adv Ther 2012; 29: 579-589.

14 Takamura E, Tsubota K, Watanabe H, Ohashi Y. Diquafosol Ophthalmic Solution Phase 3 Study Group. A randomised, double-masked comparison study of diquafosol versus sodium hyaluronate ophthalmic solutions in dry eye patients. Br J Ophthalmol 2012; 96: 1310-1315.

15 Yamaguchi M, Nishijima T, Shimazaki J, Takamura E, Yokoi N, Watanabe $\mathrm{H}$ et al. Clinical usefulness of diquafosol for real-world dry eye patients: a prospective, open-label, noninterventional, observational study. Adv Ther 2014; 31: 1169-1181.
16 Li Y, Kuang K, Yerxa B, Wen Q, Rosskothen H, Fischbarg J. Rabbit conjunctival epithelium transports fluid, and $\mathrm{P} 2 \mathrm{Y}_{2}$ receptor agonists stimulate $\mathrm{Cl}^{-}$and fluid secretion. $A m \mathrm{~J}$ Physiol Cell Physiol 2001; 281: C595-C602.

17 Murakami T, Fujihara T, Horibe Y, Nakamura M. Diquafosol elicits increases in net $\mathrm{Cl}^{-}$transport through $\mathrm{P}_{2} \mathrm{Y}_{2}$ receptor stimulation in rabbit conjunctiva. Ophthalmic Res 2004; 36: 89-93.

18 Yokoi N, Bron A, Tiffany J, Brown N, Hsuan J, Fowler C. Reflective meniscometry: a non-invasive method to measure tear meniscus curvature. Br J Ophthalmol 1999; 83: 92-97.

19 Oguz H, Yokoi N, Kinoshita S. The height and radius of the tear meniscus and methods for examining these parameters. Cornea 2000; 19: 497-500.

20 Yokoi N, Bron AJ, Tiffany JM, Kinoshita S. Reflective meniscometry: a new field of dry eye assessment. Cornea 2000; 19: S37-S43.

21 Yokoi N, Bron AJ, Tiffany JM, Maruyama K, Komuro A, Kinoshita S. Relationship between tear volume and tear meniscus curvature. Arch Ophthalmol 2004; 122: 1265-1269.

22 Fox RI, Robinson CA, Curd JG, Kozin F, Howell FV. Sjögren's syndrome. Proposed criteria for classification. Arthritis Rheum 1986; 29: 577-585.

23 Koh S, Watanabe H, Hosohata J, Hori Y, Hibino S, Nishida K et al. Diagnosing dry eye using a blue-free barrier filter. Am J Ophthalmol 2003; 136: 513-519.

24 Lemp MA. Report of the National Eye Institute/Industry Workshop on clinical trials in dry eyes. CLAO J 1995; 21: 221-232.

25 van Bijsterveld OP. Diagnostic tests in the Sicca syndrome. Arch Ophthalmol 1969; 82: 10-14.

26 Yokoi N, Komuro A. Non-invasive methods of assessing the tear film. Exp Eye Res 2004; 78: 399-407.

27 Tsubota K, Xu KP, Fujihara T, Katagiri S, Takeuchi T. Decreased reflex tearing is associated with lymphocytic infiltration in lacrimal glands. J Rheumatol 1996; 23: 313-320.

28 Tsubota K, Kaido M, Yagi Y, Fujihara T, Shimmura S. Diseases associated with ocular surface abnormalities: the importance of reflex tearing. Br J Ophthalmol 1999; 83: 89-91.

29 Chen Q, Zhang X, Cui L, Huang Q, Chen W, Ma H et al. Upper and lower tear menisci in Sjögren's syndrome dry eye. Invest Ophthalmol Vis Sci 2011; 52: 9373-9378.

30 Qiu X, Gong L, Lu Y, Jin H, Robitaille M. The diagnostic significance of Fourier-domain optical coherence tomography in Sjögren syndrome, aqueous tear deficiency and lipid tear deficiency patients. Acta Ophthalmol 2012; 90: e359-e366.

31 Ibrahim OM, Dogru M, Kawashima S, Wakamatsu TH, Tsubota K, Fujishima H. Visante optical coherence tomography and tear function test evaluation of cholinergic treatment response in patients with Sjögren syndrome. Cornea 2013; 32: 653-657.

32 Cowlen MS, Zhang VZ, Warnock L, Moyer CF, Peterson WM, Yerxa BR. Localization of ocular $\mathrm{P}_{2} \mathrm{Y}_{2}$ receptor gene expression by in situ hybridization. Exp Eye Res 2003; 77: 77-84.

33 Caffery B, Simpson T, Wang S, Bailey D, McComb J, Rutka J et al. Rose bengal staining of the temporal conjunctiva differentiates Sjögren's syndrome from keratoconjunctivitis sicca. Invest Ophthalmol Vis Sci 2010; 51: 2381-2387.

34 Pflugfelder SC, Huang AJ, Feuer W, Chuchovski PT, Pereira IC, Tseng SC. Conjunctival cytologic features of primary Sjögren's syndrome. Ophthalmology 1990; 97: 985-991. 
35 Takamura E, Takano H, Yoshino K, Negoro K, Tsubota K, Kobayashi T. Quantitative cellular evaluation of conjunctival squamous metaplasia in the dry eye patient. Adv Exp Med Biol 1994; 350: 535-538.

36 Jones DT, Monroy D, Ji Z, Pflugfelder SC. Alterations of ocular surface gene expression in Sjögren's syndrome. Adv Exp Med Biol 1998; 438: 533-536.

37 Hirai N, Kawasaki S, Tanioka H, Connon CJ, Yamasaki K, Yokoi $\mathrm{N}$ et al. Pathological keratinisation in the conjunctival epithelium of Sjögren's syndrome. Exp Eye Res 2006; 82: 371-378.

38 Koh S, Ikeda C, Takai Y, Watanabe H, Maeda N, Nishida K. Long-term results of treatment with diquafosol ophthalmic solution for aqueous-deficient dry eye. Jpn J Ophthalmol 2013; 57: 440-446.

39 Shimazaki-Den S, Iseda H, Dogru M, Shimazaki J. Effects of diquafosol sodium eye drops on tear film stability in short BUT type of dry eye. Cornea 2013; 32: 1120-1125.
40 Takeshita T. Effect of diquafosol sodium ophthalmic solution for dry eye after cataract surgery. Jpn J Clin Ophthalmol 2013; 67: 327-330.

41 Mori Y, Nejima R, Masuda A, Maruyama Y, Minami K, Miyata $\mathrm{K}$ et al. Effect of diquafosol tetrasodium eye drop for persistent dry eye after laser in situ keratomileusis. Cornea 2014; 33: 659-662.

42 Toda I, Ide T, Fukumoto T, Ichihashi Y, Tsubota K. Combination therapy with diquafosol tetrasodium and sodium hyaluronate in patients with dry eye after laser in situ keratomileusis. Am J Ophthalmol 2014; 157: 616-622.

43 Arita R, Suehiro J, Haraguchi T, Maeda S, Maeda K, Tokoro H et al. Topical diquafosol for patients with obstructive meibomian gland dysfunction. Br J Ophthalmol 2013; 97: 725-729.

44 Nakamura S, Kinoshita S, Yokoi N, Ogawa Y, Shibuya M, Nakashima $\mathrm{H}$ et al. Lacrimal hypofunction as a new mechanism of dry eye in visual display terminal users. PLoS One 2010; 5: e11119. 